\title{
Cochrane
}

Library

Cochrane Database of Systematic Reviews

\section{Anticholinergic burden (prognostic factor) for prediction of dementia or cognitive decline in older adults with no known cognitive syndrome (Protocol)}

Quinn TJ, Myint PK, McCleery J, Taylor-Rowan M, Stewart C

Quinn TJ, Myint PK, McCleery J, Taylor-Rowan M, Stewart C.

Anticholinergic burden (prognostic factor) for prediction of dementia or cognitive decline in older adults with no known cognitive syndrome.

Cochrane Database of Systematic Reviews 2020, Issue 2. Art. No.: CD013540.

DOI: 10.1002/14651858.CD013540.

www.cochranelibrary.com

Anticholinergic burden (prognostic factor) for prediction of dementia or cognitive decline in older adults with no

Copyright @ 2020 The Cochrane Collaboration. Published by John Wiley \& Sons, Ltd. 
TABLE OF CONTENTS

HEADER 1

ABSTRACT

BACKGROUND

OBJECTIVES

METHODS

ACKNOWLEDGEMENTS

REFERENCES

APPENDICES

CONTRIBUTIONS OF AUTHORS

DECLARATIONS OF INTEREST

SOURCES OF SUPPORT 
[Prognosis Protocol]

\section{Anticholinergic burden (prognostic factor) for prediction of dementia or cognitive decline in older adults with no known cognitive syndrome}

Terry J Quinn ${ }^{1}$, Phyo K Myint² ${ }^{2}$ Jenny McCleery³, Martin Taylor-Rowan¹, Carrie Stewart ${ }^{4}$

1Institute of Cardiovascular and Medical Sciences, University of Glasgow, Glasgow, UK. 2Division of Applied Health Sciences, School of Medicine, Medical Sciences and Nutrition, University of Aberdeen, Aberdeen, UK. ${ }^{3}$ Oxford Health NHS Foundation Trust, Banbury, UK.

${ }^{4}$ ACER, IAHS, University of Aberdeen, Aberdeen, UK

Contact address: Terry J Quinn, Institute of Cardiovascular and Medical Sciences, University of Glasgow, New Lister Campus, Glasgow Royal Infirmary, Glasgow, G4 0SF, UK.Terry.Quinn@glasgow.ac.uk, tjq1t@clinmed.gla.ac.uk.

Editorial group: Cochrane Dementia and Cognitive Improvement Group.

Publication status and date: New, published in Issue 2, 2020.

Citation: Quinn TJ, Myint PK, McCleery J, Taylor-Rowan M, Stewart C. Anticholinergic burden (prognostic factor) for prediction of dementia or cognitive decline in older adults with no known cognitive syndrome. Cochrane Database of Systematic Reviews 2020 , Issue 2. Art. No.: CD013540. DOI: 10.1002/14651858.CD013540.

Copyright ( 2020 The Cochrane Collaboration. Published by John Wiley \& Sons, Ltd.

\section{A B S T R A C T}

This is a protocol for a Cochrane Review (Prognosis). The objectives are as follows:

Primary objective: to assess whether ACB in older adults is a prognostic factor for future cognitive decline or dementia.

Secondary objective(s): to assess whether ACB is a prognostic factor for older adults recruited in primary care, secondary care, or community settings (with setting used as the basis for subgroup analyses); whether ACB is associated with mortality; to compare the prognostic validity of respective ACB scales; and to examine the effect of duration of exposure and duration of follow-up on the ACBdementia risk association. 


\section{B A C K G R O U N D}

\section{Description of the condition}

Cognition (or cognitive function) is the mental process of acquiring knowledge and understanding through thought, experience, and senses. It includes the domains of memory, language, attention, executive functioning, and visuospatial processing. Cognitive impairment is the disruption of functioning of any one of these domains. Cognitive function may be assessed in detail using a battery of neuropsychological tests covering multiple domains, although in clinical practice, brief assessment tools such as the Mini Mental State Examination (MMSE) or Montreal Cognitive Assessment (MoCA) are often used (Folstein 1975; Nasreddine 2005).

Dementia is a syndrome of decline in cognitive function beyond that expected from normal ageing, to an extent that interferes with usual functioning. It may affect memory, thinking, orientation, comprehension, calculation, learning capacity, language, and judgement. There are a variety of internationally accepted diagnostic criteria for dementia, the most widely used of which are included in the World Health Organization International Classification of Diseases (ICD) and the American Psychiatric Association Diagnostic and Statistical Manual of Mental Disorders (DSM). The most recent iteration of the DSM (DSM-5) refers to 'major neurocognitive disorder' instead of dementia. The labels of 'dementia' or 'major neurocognitive disorder' encompass a variety of pathologies, with specific diagnostic criteria also available for pathologically defined dementia subtypes, such as the National Institute of Neurological and Communicative Disorders and Stroke and the Alzheimer's Disease and Related Disorders Association (NINCDS-ADRDA) criteria for dementia due to Alzheimer's dementia (AD) (McKhann 1984; McKhann 2011); McKeith criteria for Lewy body dementia (McKeith 2005); Lund criteria for frontotemporal dementias (McKhann 2001); and the National Institute of Neurological Disorders and Stroke and the Association Internationale pour la Recherche et l'Enseignement en Neurosciences (NINDS-AIREN) criteria for vascular dementia (Román 1993).

An individual may experience a decline in cognition that is not enough to merit a label of dementia but that is more than would be expected as part of ageing. An objective cognitive impairment that is not severe enough to have a significant impact on daily activities is often referred to as a mild cognitive impairment. This is a risk factor for future dementia (Petersen 2001).

Dementia and cognitive decline are major public health issues. There are currently more than 40 million people worldwide with dementia due to $A D$, the most common subtype, and this number is projected to increase to more than 100 million by 2050 (Prince 2016). Dementia costs were estimated at USD 818,000 million in 2015 , equivalent to $1.1 \%$ of global gross domestic product. It is estimated that by 2030 , the global cost of dementia could grow to USD 2,000,000 million, which could overwhelm health and social care systems (Wimo 2017). The total cost of dementia to the UK alone is GBP 34,700 , million, of which GBP 4,900 million is paid by the National Health Service (NHS) and GBP 15,700 million is paid by social care. The remainder is paid by those living with dementia and their families, and is classified as unpaid social care or private care (Alzheimer's Society 2019).
A number of prognostic factors have been associated with the onset of dementia, including age, sex, premorbid intelligence, genetics, medical conditions (e.g. diabetes, hypertension), and lifestyle factors (e.g. physical inactivity) (Livingston 2017). Identification of prognostic factors could assist healthcare professionals in predicting outcomes for individuals with cognitive syndromes and help policymakers in planning for future population healthcare needs. Identification of modifiable prognostic factors offers potential targets for preventing or delaying the onset of cognitive decline and dementia.

\section{Description of the prognostic factor}

A prognostic factor is any measure that is associated with a future clinical outcome. The prognostic factor of interest for this review is anticholinergic burden.

A wide range of medications commonly used in older adults have anticholinergic properties. Some medications, such as oxybutynin (for overactive bladder), exert their intended effects through their anticholinergic activity. For other medications, such as amitriptyline for depression, anticholinergic activity is probably incidental to their intended mechanism of action. It is common for older adults to be taking multiple medications with anticholinergic effects (Myint 2015).

Even medications that have low anticholinergic activity individually may contribute to a significant overall anticholinergic effect if a person is taking several of them. This can be quantified as the anticholinergic burden (ACB). There is a reported relationship between ACB and various adverse health outcomes (Singh 2008). It has been suggested that exposure to high ACB is associated with cognitive decline and dementia in older adults (Fox 2014).

ACB measures are used in primary and secondary care as part of the medication review process. Such reviews are increasingly recommended for older adults. The quantification of ACB is designed to assess risk of future adverse events. Based on the $A C B$ score, clinicians may recommend reducing or replacing certain medications. Use of measures of ACB to guide treatment decisions is entering clinical practice. The most recent National Institute for Health and Care Excellence (NICE) dementia guideline recommends considering $A C B$ as a factor that may be contributing to cognitive impairment and suggests using a validated scale to measure ACB (NICE 2018). To date, ACB has been considered largely as a 'stand-alone' prognostic factor (not as part of a multifactorial prediction model).

\section{Measures of ACB}

$A C B$ can be measured using a variety of approaches. There is no consensus on which $A C B$ measures provide the most accurate and clinically useful prognostic information. Generally, ACB measures use a person's medication list and assign a score to certain medications. A cumulative total based on all prescribed medications is then calculated. Although these measures should be similar, they have been developed using different methods and so include differing medications and assign differing scores to these medications.

Our literature scoping suggests that 10 tools to measure ACB have been published. In a large population cohort (UK Biobank) (Hanlon 2019), it was reported that ACB was strongly associated with future adverse health outcomes regardless of which ACB measure is used. 
However, at the individual patient level there was substantial variability in the ACB score generated by each measure. The overlap between measures is limited, and so any prognostic review should be completed at the individual scale level in addition to trying to create summary estimates for all ACB measures.

Prior to adoption in clinical practice, there should be a comprehensive assessment of the available literature to describe whether ACB is a true prognostic factor, particularly after adjusting for other dementia risk factors that may also be associated with anticholinergic prescribing. If $A C B$ is a prognostic factor, the strength of the association and the quality of the supporting evidence should also be described. Looking at the prognostic properties of each ACB measure may assist in choosing a preferred scale.

\section{Why is it important to do this review?}

This increasing clinical interest in ACB is accompanied by a growth in the research literature on $A C B$ as a prognostic factor. However, results from individual studies of $A C B$ and cognition or dementia are conflicting, and not all published papers have followed best practice in design, conduct, or reporting. There is thus uncertainty around the clinical utility of $A C B$ and consequently inconsistency in clinical practice and guideline recommendations. In this systematic review, we aim to estimate the prognostic utility (adjusted and unadjusted) of different $A C B$ measures for predicting dementia or cognitive decline in a cognitively healthy older adult population and to assess the quality of the supporting evidence. The review has the potential for an immediate impact on practice, particularly if ACB measures have demonstrably different prognostic utility.

Our scoping suggests that certain measures will have sufficient published data to allow for a meta-analytical approach to describing summary prognostic utility. For other measures, our scoping suggests there may be no relevant papers. This lack of evidence would still represent an important finding, particularly in the context of increasing enthusiasm for the clinical application of these measures.

It will take a randomised controlled trial to definitively show whether using $A C B$ measures can guide deprescribing of anticholinergic medications in older adults and whether such an intervention is clinically effective, cost-effective, and safe. Our review may inform such a trial by suggesting the preferred method for quantifying $A C B$. Whilst we await this definitive evidence of the utility of the ACB concept, our proposed review will offer a critical summary of the evidence around $A C B$ as a prognostic factor.

\section{O B JECTIVES}

Primary objective: to assess whether $A C B$ in older adults is a prognostic factor for future cognitive decline or dementia.

Secondary objective(s): to assess whether ACB is a prognostic factor for older adults recruited in primary care, secondary care, or community settings (with setting used as the basis for subgroup analyses); whether ACB is associated with mortality; to compare the prognostic validity of respective $A C B$ scales; and to examine the effect of duration of exposure and duration of follow-up on the ACBdementia risk association.

\section{METHODS}

We will follow best practice in design, conduct, and reporting of our prognosis review as detailed in the Cochrane Handbook for Systematic Reviews of Interventions (Cochrane 2019). The review will be supported by the Cochrane Prognostic Methods Group, partners within the Cochrane Mental Health and Neuroscience Network, and the UK National Institute for Health Research Complex Reviews Support Unit (NIHR CRSU).

We used the PICOT (Patient/Problem; Intervention; Comparison; Outcome; Timing) system to design our review question (Schardt 2007). As recommended by the Cochrane Prognosis Methods Group, we will follow MOOSE (meta-analysis of observational studies in epidemiology) guidelines, complemented by REMARK (Reporting Recommendations for Tumor Marker Prognostic Studies), to ensure that our review is designed, conducted, and reported in keeping with best practice recommendations (Riley 2019).

\section{PICOTS}

Population Older adults (mean age of 50 years and above) free of cognitive impairment at baseline

Interventions Anticholinergic burden as measured by any validated ordinal anticholinergic burden scale

Comparators (covariates of in- Age, sex, and comorbidity terest)

Outcomes Incident dementia or cognitive function (multidomain)

Type of study Longitudinal, observational cohort/case-control

Timing and setting 


\section{Selection criteria}

\section{Types of studies}

We will include prospective and retrospective longitudinal cohort and case-control observational studies. We will not include crosssectional studies, as it is not possible to determine prognosis from this design. We will not include prospective case studies, defined here as having fewer than 20 participants. We will exclude studies that are published only as abstracts or posters at conferences, as these have not undergone peer review.

\section{Types of participants}

We will include any studies that recruited older adults (as defined by the authors, but with minimum median/mean age 50 years at baseline) who were free of any known cognitive diagnosis (mild cognitive impairment, dementia, delirium) at time of recruitment and at time of application of the ACB measure. We will not exclude studies that did not assess cognition at baseline; however, where a mixed population was recruited, we will only include the study in the review if the prevalence of dementia is less than $7 \%$ in a community sample (similar to general population prevalence in unselected older adults). We will also include studies that recruited mixed populations in a hospital setting, where the prevalence of dementia may be higher, provided that it is conducted in an unselected sample. A separate prognosis review is planned to look at the prognostic utility of $A C B$ in people living with cognitive syndromes. This future review will complement the findings of the present review by expanding our findings to ACB risk for cognitive decline in those who already demonstrate cognitive issues. We will make no other restriction based on comorbidity or polypharmacy, but will record these factors in our data extraction. We will assess whether comorbid conditions that are associated with dementia (depression, stroke, other neurological diseases) were measured and consider any potential impact of this in our 'Risk of bias' assessment. We will include studies conducted in specific patient subgroups, such as Parkinson's disease or stroke, provided they meet our other inclusion criteria. We believe these criteria will give a population representative of the people in whom ACB may be used in clinical practice.

\section{Index prognostic factor}

The prognostic factor of interest is anticholinergic burden. We will include any study that used an ordinal scale that purports to measure cumulative exposure to medications with anticholinergic properties. Scales need not be described as validated for prediction of cognitive outcomes. Previously identified scales are listed in Appendix 1.

Different approaches to quantifying ACB have been used. Some scales sum ordinal scores for each relevant drug to give a continuous measure; others create a summary ordinal hierarchical measure that scores, for example, 0 to 3 or 0 to 4 based on the cumulative anticholinergic exposure. Most scales define thresholds of 'low' and 'high' burden. As our focus in this review is on the extent of anticholinergic burden, we will not include studies that assess anticholinergic exposure via a simple dichotomised present/absent scale.

Some ACB measures were developed specifically to predict dementia, whilst others were developed to predict other adverse events, including death. Anticholinergic activity should be an objective drug effect, and so we will include any ACB measure, not just those developed for cognitive outcomes.

We have not chosen a particular measure of primary interest as there is no consensus on the preferred measure, and there is substantial heterogeneity in clinical practice. However, if the Drug Burden Index (DBI) scale is utilised, we will only include data if $A C B$ data are reported separately.

\section{Comparator prognostic factors}

We are interested in the value of $A C B$ as a prognostic factor over and above other prognostic factors that may be common in this population. Hence, whilst we will include studies that only assessed the unadjusted $A C B$ prognosis, we will also evaluate the prognostic effect of $A C B$ after adjustment for core variables identified as fundamental to the putative link between ACB and dementia. These variables were selected on the basis of a Delphi discussion between the review authors and a wider multicentre collaborative working in the field of $A C B$ research. The chosen core variables are: age, sex, and comorbidity. We recognise that comorbidity may be described in various ways. We will accept any classification that the original study authors define as a measure of comorbidity, including measures that offer quantitative data, for example number of medications, number of medical conditions listed, or formal measures such as the Charlson Index. We will assess use of additional adjustments in our 'Risk of bias' assessment.

\section{Outcome measures}

Primary outcome: We will include any study that assesses incident dementia or cognitive decline (i.e. change on a measure of cognitive function) as an outcome. We will accept any validated diagnostic criteria for dementia.

For the outcome of cognitive decline, we will accept any multidomain cognitive assessment tool that is validated for the direct assessment of cognition. We will not include papers that only measure a single cognitive domain.

Secondary outcome: We will include overall survival and dementia-free survival as secondary outcomes in studies that measure cognitive outcomes or incidence of dementia.

Timing: We will accept assessment for dementia or cognitive decline at any time greater than or equal to one year following baseline $A C B$ assessment so as to mitigate the risk of reverse causality between any observed risk associations. We anticipate that most studies will have a follow-up of at least one year to allow time for outcomes to develop. If possible, we will collate outcomes at common time periods, for example one year, two years, three years.

Setting: We will include studies where initial recruitment was in primary care, secondary care, and community settings. We will define primary care as settings in which the patient self-presents to a non-specialist service, such as general practice. We will define secondary care as any settings where patients are referred for expert care, including general hospitals and more specialist settings. We will define community settings as settings in which the cohort is completely unselected, that is 'population screening'. Prevalence of dementia is likely to be lower in such settings than in secondary or primary care. As this review is focused on prognosis 
in people who are cognitively well, we will not include studies conducted in care-home settings where the prevalence of cognitive syndromes is substantial.

\section{Search methods for identification of studies}

\section{Electronic searches}

As reporting of prognostic factor studies is variable, it can be challenging to identify all relevant studies. We will adopt the procedure proposed by Geersing 2012 to maximise our ability to identify relevant prognostic studies. Specifically, as we are searching for one prognostic factor, we will not adopt any specific search filter, but will instead adopt a search that combines our prognostic factor (ACB) with the disease outcome (dementia/ cognitive impairment). Search strategy can be seen in Appendix 2.

We will search the following databases from inception year to present date: the Cochrane Dementia and Cognitive Improvement Group Specialised Register, Ovid Medline, Ovid Embase, APA (American Psychological Association) PsycInfo, EBSCO's (Elton B. Stephens Co.) CINAHL (Cumulative Index to Nursing and Allied Health Literature), and ISI Web of Science Core Collection.

\section{Searching other resources}

We will supplement this with handsearches of all included studies and identified systematic reviews and will contact study authors for missing data where required.

\section{Data collection and analysis}

\section{Selection of studies}

We will use Covidence systematic review software (Covidence) to identify relevant studies. Two review authors will independently screen studies identified via our search method. Titles and abstracts will be screened in the first instance, with the full text of potentially relevant studies then accessed to determine if the study meets our inclusion criteria. In case of disagreement, a third review author will act as arbiter and make the final decision on study inclusion/exclusion. We will illustrate the results of the search using a PRISMA flow chart (Moher 2009).

\section{Data extraction and management}

Two review authors will extract the data to a piloted pro forma based on the CHARMS-PF (CHecklist for critical Appraisal and data extraction for systematic Reviews of prediction Modelling Studies, adapted for prognostic factors) template (Riley 2019). We will contact authors for missing data where required. We will select two studies, Richardson 2018; Gray 2015, with which to trial our data extraction pro forma. We will extract all data onto a standard form containing the following information.

Contents of data extraction pro forma

\begin{tabular}{ll}
\hline Extracted information & Included details \\
\hline General information & Author, title, source, publication date, language, related or duplicate publications \\
\hline Source of data & $\begin{array}{l}\text { Cohort (retrospective or prospective data collection), case-control, randomised trial, or secondary } \\
\text { analysis of registry data }\end{array}$ \\
\hline Participant information & $\begin{array}{l}\text { Participant eligibility and recruitment method (e.g. consecutive or other recruitment, number of } \\
\text { centres, inclusion and exclusion criteria); participant demographics (e.g. age, sex); details of ongo- } \\
\text { ing treatments/medications; study dates; country of recruitment; setting (using our definitions of } \\
\text { primary, secondary, and community settings) }\end{array}$
\end{tabular}

Prognostic factor

Definition and method of measurement of prognostic factor. We do not expect duration of exposure (pre- or post-study commencement) to be regularly recorded; however, where possible, we will record timing of prognostic factor measurement (number of weeks participants have been on the ACB drugs prior to baseline assessment); where data are available we will also collect duration of exposure during the study.

Outcomes to be predicted

Definition and method of measurement of outcome; time of outcome ascertainment, or summary of duration of follow-up, overall survival, and dementia-free survival

Adjustment for other prognos- List of all the covariates that were adjusted for in any regression model tic factors (covariates)

$\begin{array}{ll}\text { Sample size } & \begin{array}{l}\text { Number of participants and number of outcomes/events; how missing data were handled (e.g. } \\ \text { complete-case analysis, imputation, or other methods) }\end{array}\end{array}$

Reported results

We will record incidence of dementia and cognitive decline. Data on incident dementia are likely to be in the form of hazard ratios. Where possible, we will extract estimates and corresponding confidence intervals from each included paper. Change in validated cognitive assessment data may be presented as continuous data or as events with hazard ratios (e.g. time till cognitive score declines 
below a particular value). We will also record overall survival (including duration of follow-up) and dementia-free survival (including duration of follow-up).

\section{Assessment of methodological quality}

Two review authors will independently use the QUIPS (Quality in Prognosis Studies) checklist (Hayden 2012), assessing the included studies across the domains of: study participation; study attrition; prognostic factor measurement; outcome measurement; adjustment for covariates; statistical analyses and reporting. We will take the QUIPS anchoring statements but modify content to suit our review topic based on consensus within the review author team. We will judge each domain as low risk of bias, moderate risk of bias, or high risk of bias.

Specific considerations for this review: We will assess issues with risk of bias around the following.

- Study participation: We will consider whether the method of recruitment is at risk of selection bias (e.g. consecutive recruitment versus convenience sample) and if there is adequate reporting of comorbidities and demographics (age and sex). If either a convenience sample was used or there was inadequate reporting of comorbidities/demographics, we will assign a moderate risk of bias.

- Attrition: We anticipate that most studies will utilise databases, hence attrition will be less of an issue. We will therefore focus on reporting of, and methods for dealing with, missing data. We will assign a moderate risk of bias if no analysis was carried out to evaluate if participants with missing data differed in baseline ACB score compared to those with full data.

- Prognostic factor measurement: We will consider how medication data was obtained. If medication was not established via at least two methods and capable of establishing non-prescription medications taken, along with duration of exposure and adherence, we will assign a moderate risk of bias. If repeated ACB measurements were not made over time for studies with a greater than one-year follow-up duration, we will assign a high risk of bias. We anticipate that some studies may utilise validated ACB scales but tweak these scales, for instance to incorporate dosage into the anticholinergic calculation. We will not consider utilisation of ACB scales as part of the 'Risk of bias' assessment, as it is the purpose of the review to establish which ACB scales have the greatest prognostic accuracy.

- Outcome measurement: We will consider the method utilised for dealing with missing data in relation to the outcome. If 'last diagnosis carried forward' was used when final outcome data were not available, we will assign a high risk of bias. We will assess whether the outcome was established via a clinical follow-up or was reliant upon database diagnoses. If outcome was reliant upon database diagnoses alone, we will assign a moderate risk of bias. We will also assess if the outcome was determined without knowledge of the prognostic factor, and assign a high risk of bias if there was no blinding to outcome.

- Covariates: We will assess whether studies adjusted for age, sex, and comorbidities as a minimum. If these covariates were not adjusted for, we will assign a high risk of bias. Assessment for comorbidities should control for at least three comorbidities that cover both physical and psychiatric domains; failure to do so will result in a rating of moderate risk of bias.
- Reverse causation: We will evaluate studies regarding perceived risk that anticholinergic drug use was prescribed for treatment of early signs of dementia. If a study does not restrict ACB measurement to 12 months before dementia onset, we will assign a high risk of bias.

- Statistical analysis: We will evaluate how the analysis was conducted. We will assign a high risk of bias if: a multivariate analysis was not conducted; if the analysis was not appropriately powered based on the ' 10 events per covariate'; if the method for selecting covariates for inclusion in a multivariate model was based on $P$ values in a univariate analysis without incorporation of prior knowledge of relevant associations into selection; if the method of analysis was consistent with the stated protocol; and if the reported results are consistent with the stated method of analysis. We will assign a moderate risk of bias if relevant assumptions were not checked.

Specific issues of consideration in each area were decided upon via discussion amongst the review authors.

Discussing reporting deficiencies: Prognosis research is often confounded by poor reporting and possible publication bias. We will supplement our 'Risk of bias' assessment with a narrative discussion of reporting issues, highlighting when missing information may affect results. Prognostic factor studies often do not register protocols, increasing the risk that not all studies (published and unpublished) will be identified, and there is a risk of small-study effects (in which smaller studies with higher odds ratios are more likely to be published than smaller studies with non-significant odds ratios), which can bias meta-analyses (Peat 2014; Riley 2019). We will use sensitive search filters for the disease (dementia) and the prognostic factor (ACB burden) without any specific filter for prognostic research to increase retrieval, and will also examine the likelihood of small-study effects in our review by generating a funnel plot.

\section{Data synthesis}

To investigate the $A C B$ association with our primary and secondary outcomes, we will evaluate ACB scores as an ordinal, hierarchical variable. We will offer summary estimates for each ACB tool, and as an exploratory analysis (if data allow) we will also describe a summary estimate including all ACB measures. We will pool data in three separate ways. In the first instance, we will pool data obtained from unadjusted analyses. In the second instance, we will pool data from fully adjusted analyses, provided age, sex, and comorbidity were controlled for as a minimum. And as a final exploratory analysis, we will pool data from the most adjusted model (i.e. the model adjusting for the greatest number of variables) presented. We will pool odds, hazard, risk ratios and correlations separately. Where data are not available, we will attempt to estimate data based on methods suggested by Tierney 2007. Where data are sufficiently similar to permit pooling, we will use a random-effects approach given our expectation of high heterogeneity between studies. We will use Comprehensive MetaAnalysis software (Borenstein 2013) to conduct all meta-analyses. 
If time and data allow, we will attempt comparative analyses of the differing ACB measures. We will work with the team from the NIHR CRSU on this aspect of the analysis.

We will assess exposure including exposure before enrolment into the study and exposure during the course of the study. Where data allow, we will create categories of $<1$ year, 1 to 5 years, and $>$ 5 years. In a sensitivity analysis, we will remove studies with $<1$ year exposure. If there are sufficient papers, we will run a subgroup analysis looking at 1 to 5 years and $>5$ years individually.

We will assess duration of follow-up. Where data allow, we will create categories of $<1$ year, 1 to 5 years, and $>5$ years. In a sensitivity analysis, we will remove studies with $<1$ year followup. If there are sufficient papers, we will run a subgroup analysis looking at 1 to 5 years and $>5$ years individually.

We will also conduct a subgroup analysis via setting. These additional outcomes were decided upon through discussion amongst the review authors.

Finally, we will conduct a sensitivity analysis restricted to studies that scored as low risk of bias in all respective domains.

\section{Investigation/description of heterogeneity}

We will investigate and describe statistical heterogeneity via the $1^{2}$ statistic (I2 greater than 30\% represents moderate heterogeneity; $I^{2}$ greater than $75 \%$ considerable heterogeneity) (Higgins 2003). Where data allow, we will conduct a meta-regression based on study recruitment setting (primary versus secondary care versus community care), comorbidity, and polypharmacy in our analysis of heterogeneity.

\section{Grading the evidence}

We will use the GRADE approach to evaluate our overall confidence in the results. We will adapt the GRADE approach to suit prognosis research in a fashion consistent with Huguet 2013. Specifically, we will evaluate reported evidence in the following eight areas.

1. Phase of investigation: Phase 3 explanatory studies derived from bespoke cohort study designs that seek to explain the mechanisms behind an underlying association between ACB and dementia/cognition will be considered a high level of evidence. Phase 2 explanatory studies that seek to confirm an independent association between ACB and dementia/cognition will be treated as moderate evidence, and hypothesis generating phase 1 explanatory studies will be treated as weak evidence for any association between ACB and dementia/cognition.

2. Study limitations: We will use the previously described QUIPS tool to evaluate the overall risk of bias of included studies. Our GRADE judgement will be based upon the overall quality of the evidence, i.e. if most (> 50\%) included studies are considered to be at high risk of bias in their reported association between $A C B$ and dementia/cognition, we will downgrade the evidence accordingly.
3. Inconsistency: We will downgrade the evidence if associations between ACB and dementia/cognition are heterogeneous (i.e. the reported odds/hazard ratios fall either side of 1.0 on a forest plot); if the $I^{2}$ statistic is substantial (i.e. $75 \%$ ); and if the $P$ value is low for the test of the null hypothesis that all studies in a metaanalysis have the same underlying magnitude effect.

4. Indirectness: We will downgrade the studies where their investigation does not fully match with our broader review question. We will consider two areas of indirectness when judging if evidence should be downgraded on this basis:

a. if the population in the included studies only represents a subset of the population of interest (e.g. if only very old, i.e. $>80$ years, were assessed);

b. if the outcome investigated in the included studies is overly restricted, e.g. if the included studies explored only the association between ACB and Alzheimer's dementia, then the evidence for the association between ACB and all-cause dementia would be downgraded for indirectness.

5. Imprecision: We will downgrade the evidence if there are insufficient numbers in the meta-analysis or if the confidence intervals are wide. We will not set an absolute value, but will assess this in the context of effect size and minimally important clinical difference. If a meta-analysis is not possible, we will evaluate imprecision on the basis of study power and reported confidence intervals within individual studies. We will also downgrade the evidence if estimation of hazard/odds ratios using summary data employing methods described by Tierney 2007 is required.

6. Publication bias: Due to inherent issues regarding publication bias in prognostic research, we will adopt the default position that publication bias is likely and downgrade the evidence unless our assessment of publication bias provides significant evidence to the contrary (i.e. a symmetrically distributed funnel plot, and evidence that the prognostic factor has been investigated in a large number of cohort studies).

7. Effect size: We will upgrade our confidence in the effect estimate if the effect size is moderate to large (e.g. a hazard ratio of 2.5 or above).

8. Exposure-response gradient: We will upgrade our confidence in the effect estimate if there is evidence (via subgroup analysis) that a longer duration of ACB is associated with an increased risk of dementia/cognitive decline. Similarly, we will upgrade the evidence if there is an incremental increase in effect size with increasing $\mathrm{ACB}$.

\section{ACK N OWLEDGEMENTS}

We would like to thank our funders for supporting this work. We would like to thank the CDCIG Information specialist for helping to develop the MEDLINE search strategy.

We would like to thank peer reviewers Angela Aldin, Chris Fox and Noll Campbell for their feedback and comments 


\section{R E F E E N CE S}

\section{Additional references}

\section{Alzheimer's Society 2019}

Alzheimer's Society. Annual Report Overview 2018/19. https:// www.alzheimers.org.uk/about-us/policy-and-influencing/ dementia-scale-impact-numbers.

\section{Borenstein 2013 [Computer program]}

Borenstein, M, Hedges, L, Higgins, J, \& Rothstein, $H$. Comprehensive Meta-Analysis Version 3. Englewood, $\mathrm{NJ}$ : Biostat, 2013.

\section{Cochrane 2019}

Higgins JPT, Thomas J, Chandler J, Cumpston M, Li T, Page MJ, et al (editors). Cochrane Handbook for Systematic Reviews of Interventions version 6.0 (updated July 2019).. Cochrane, 2019. Available from www.training.cochrane.org/handbook..

\section{Covidence [Computer program]}

Veritas Health Innovation. Covidence systematic review software. Melbourne, Australia: Veritas Health Innovation.

\section{Folstein 1975}

Folstein MF, Folstein SE, McHugh PR. "Mini-mental state". A practical method for grading the cognitive state of patients for the clinician. Journal of Psychiatric Research 1975;12:189-98.

\section{Fox 2014}

Fox C, Smith T, Maidment I, Chan WY, Bua N, Myint PK, et al. Effect of medications with anti-cholinergic properties on cognitive function, delirium, physical function and mortality: a systematic review. Age Ageing 2014;43:604-15.

\section{Geersing 2012}

Geersing GJ, Bouwmeester W, Zuithoff P, Spijker R, Leeflang M, Moons KG. Search filters for finding prognostic and diagnostic prediction studies in Medline to enhance systematic reviews. PLOS ONE 2012;7:e32844.

\section{Gray 2015}

Gray SL, Anderson ML, Dublin S, Hanlon JT, Hubbard R, Walker R, et al. Cumulative use of strong anticholinergics and incident dementia: a prospective cohort study. JAMA International Medicine 2015;175:401-7.

\section{Hanlon 2019}

Hanlon P, Quinn TJ, Gallacher KI, Myint PK, Jani BD, Nicholl BI, et al. Assessing risks of polypharmacy involving medications with anticholinergic properties. Annals of Family Medicine (in press).

\section{Hayden 2012}

Hayden JA, van der Windt DA, Cartwright JL, Côté P, Bombardier C. Assessing bias in studies of prognostic factors. Annals of International Medicine 2012;158(4):280-6.

\section{Higgins 2003}

Higgins JPT, Thompson SG, Deeks JJ, Altman DG. Measuring inconsistency in meta-analyses. The BMJ 2003;327:557-60.

\section{Huguet 2013}

Huguet A, Hayden JA, Stinson J, McGrath PJ, Chambers CT, Tougas ME, et al. Judging the quality of evidence in reviews of prognostic factor research: adapting the GRADE framework. Systematic Reviews 2013;2:71.

\section{Livingston 2017}

Livingston G, Sommerlad A, Orgeta V, Costafreda SG, Huntley J, Ames $D$, et al. Dementia prevention, intervention, and care. Lancet 2017;390:2673-734.

\section{McKeith 2005}

McKeith IG, Dickson DW, Lowe J, Emre M, O'Brien JT, Feldman H, et al. Diagnosis and management of dementia with Lewy bodies: third report of the DLB Consortium. Neurology 2005;65:1863-72.

\section{McKhann 1984}

McKhann G, Drachman D, Folstein M, Katzman R, Price D, Stadlan EM. Clinical diagnosis of Alzheimer's disease: report of the NINCDS-ADRDA Work Group under the auspices of Department of Health and Human Services Task Force on Alzheimer's Disease. Neurology 1984;34:939-44.

\section{McKhann 2001}

McKhann GM, Albert MS, Grossman M, Miller B, Dickson D, Trojanowski JQ. Work Group on Frontotemporal dementia and Pick's disease. Clinical and pathological diagnosis of frontotemporal dementia: report of the Work Group on Frontotemporal Dementia and Pick's Disease. Archives of Neurology 2001;58:1803-9.

\section{McKhann 2011}

McKhann GM, Knopman DS, Chertkow H, Hyman BT, Jack CR Jr, Kawas $\mathrm{CH}$, et al. The diagnosis of dementia due to Alzheimer's disease: recommendations from the National Institute on Aging - Alzheimer's Association work group on diagnostic guidelines for Alzheimer's disease. Alzheimer's \& Dementia 2011;7:263-9.

\section{Moher 2009}

Moher D, Liberati A, Tetzlaff J, Altman DG, PRISMA Group. Preferred reporting items for systematic reviews and meta-analyses: the PRISMA statement. PLoS Medicine 2009;6:e1000097.

\section{Myint 2015}

Myint PK, Fox C, Kwok CS, Luben RN, Wareham NJ, Khaw KT. Total anticholinergic burden and risk of mortality and cardiovascular disease over 10 years in 21,636 middle-aged and older men and women of EPIC-Norfolk prospective population study. Age Ageing 2015;44:219-25.

\section{Nasreddine 2005}

Nasreddine ZS, Phillips NA, Bédirian V, Charbonneau S, Whitehead V, Collin I, et al. The Montreal Cognitive Assessment, MoCA: a brief screening tool for mild cognitive impairment. Journal of the American Geriatric Society 2005;53:695-9.

Anticholinergic burden (prognostic factor) for prediction of dementia or cognitive decline in older adults with no known cognitive 


\section{NICE 2018}

National Institute for Health and Care Excellence. Dementia: assessment, management and support for people living with dementia and their carers. www.nice.org.uk/guidance/ng97 2018.

\section{Peat 2014}

Peat G, Riley RD, Croft P, Morley KI, Kyzas PA, Moons KGM, et al. PROGRESS Group. Improving the transparency of prognosis research: the role of reporting, data sharing, registration, and protocols. PLoS Medicine 2014;11(7).

\section{Petersen 2001}

Petersen RC, Stevens JC, Ganguli M, Tangalos EG, Cummings JL, DeKosky ST. Practice parameter: early detection of dementia: mild cognitive impairment (an evidence-based review). Report of the Quality Standards Subcommittee of the American Academy of Neurology. Neurology 2001;56:1133-42.

\section{Prince 2016}

Prince M, Comas-Herrera A, Knapp M, Guerchet M, Karagiannidou M. Improving healthcare for people living with dementia: coverage, quality and costs now and in the future. Alzheimer's Disease International (ADI): World Alzheimer Report; 2016 Sept.

\section{Richardson 2018}

Richardson K, Fox C, Maidment I, Steel N, Loke YK, Arthur A, et al. Anticholinergic drugs and risk of dementia: case-control study. The BMJ 2018;361:k1315.

\section{AP PE N D I E S}

\section{Appendix 1. Anticholinergic burden scales}

AAS: Anticholinergic activity scale

AAS-r: Revised anticholinergic activity scale

ACB: Anticholinergic burden

ACoB: Anticholinergic cognitive burden

ADS: Anticholinergic drug scale

AEC: Anticholinergic effect on cognition

AIS: Anticholinergic impregnation scale

ALS: Anticholinergic loading scale

ARS: Anticholinergic risk scale

CrAS: Clinician-rated anticholinergic scale

\section{Appendix 2. MEDLINE search strategy}

1. cholinergic antag ${ }^{\star} . t i, a b$.

2. anticholinergic ${ }^{\star} . t i, a b$.

3. anti-cholinergic ${ }^{\star}$.ti,ab.

4. cholinergic Antagonists/tu

5. Cholinergic Antagonists/ae

\section{Riley 2019}

Riley RD, Moons KGM, Snell KIE, Ensor J, Hooft L, Altman DG, et al. A guide to systematic review and meta-analysis of prognostic factor studies. The BMJ 2019;30:364:k4597.

\section{Román 1993}

Román GC, Tatemichi TK, Erkinjutti T, Cummings JL, Masdeu JC, Garcia JH, et al. Vascular dementia: diagnostic criteria for research studies. Report of the NINDS-AIREN International Workshop. Neurology 1993;43:250-60.

\section{Schardt 2007}

Schardt C, Adams MB, Owens T, Keitz S, Fontelo P. Utilization of the PICO framework to improve searching PubMed for clinical questions. BMC Medical Informatics and Decision Making 2007;15:7-16

\section{Singh 2008}

Singh S, Loke YK, Furberg CD. Inhaled anticholinergics and risk of major adverse cardiovascular events in patients with chronic obstructive pulmonary disease: a systematic review and metaanalysis. JAMA 2008;300:1439-50.

\section{Tierney 2007}

Tierney JF, Stewart LA, Ghersi D, Burdett S, Sydes MR. Practical methods for incorporating summary time-to-event data into meta-analysis. Trials 2007;8(16).

\section{Wimo 2017}

Wimo A, Guerchet M, Ali GC, Wu YT, Prina AM, Winblad B, et al. The worldwide costs of dementia 2015 and comparisons with 2010. Alzheimers Dementia 2017;1:1-7.

Anticholinergic burden (prognostic factor) for prediction of dementia or cognitive decline in older adults with no known cognitive 


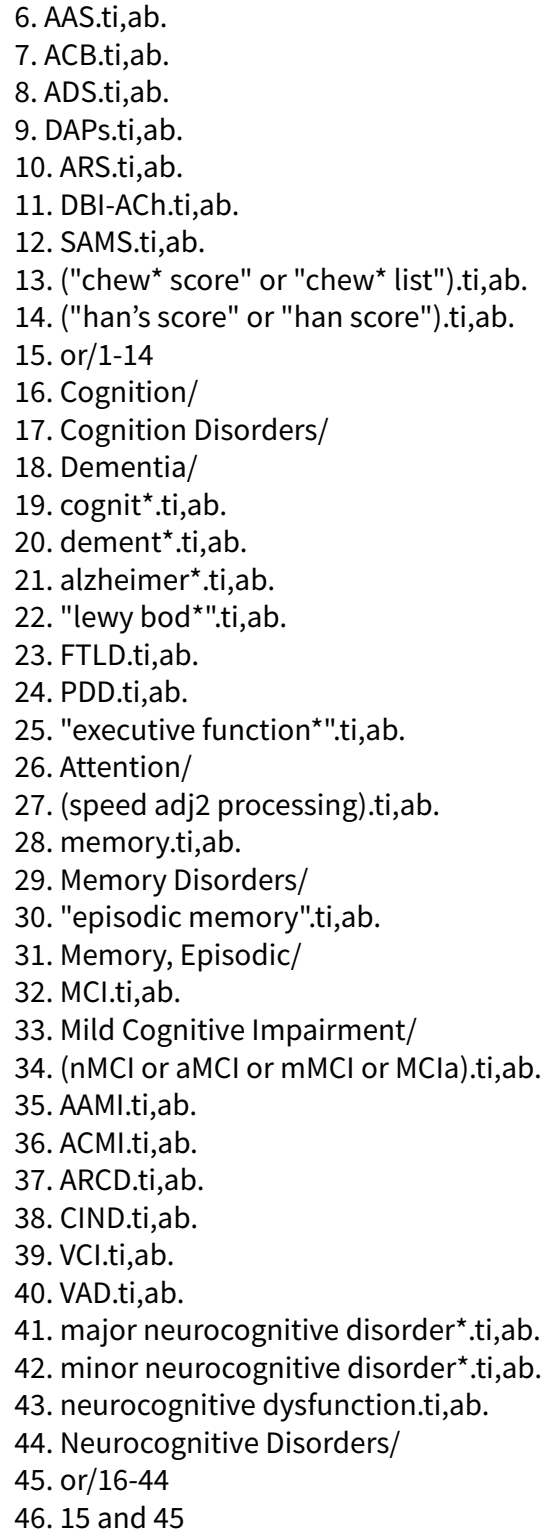

\section{CONTRIBUTIONS OF AUTHORS}

Terry Quinn conceived the idea. Martin Taylor-Rowan, Terry Quinn, and Jenny McCleery wrote the protocol. Martin Taylor-Rowan and Carrie Stewart will assess the search results (the Cochrane Dementia and Cognitive Improvement Group Information Specialist will conduct the search), select studies for inclusion and exclusion, perform data extraction and 'Risk of bias' assessment for each study. Phyo Myint provided supervision and contributed to the writing of the protocol.

\section{DECLARATIONSOF INTEREST}

Terry J Quinn: nothing to declare.

Phyo K Myint: nothing to declare.

Jenny McCleery: nothing to declare.

Martin Taylor-Rowan: nothing to declare.

Carrie Stewart: nothing to declare.

\section{SOURCES OF SUPPORT}

\section{Internal sources}

- No sources of support supplied

Anticholinergic burden (prognostic factor) for prediction of dementia or cognitive decline in older adults with no known cognitive

Copyright @ 2020 The Cochrane Collaboration. Published by John Wiley \& Sons, Ltd. 


\section{External sources}

- NIHR, UK.

This protocol was supported by the National Institute for Health Research (NIHR), via Cochrane Infrastructure funding to the Cochrane Dementia and Cognitive Improvement group. The views and opinions expressed therein are those of the authors and do not necessarily reflect those of the Systematic Reviews Programme, NIHR, National Health Service or the Department of Health 